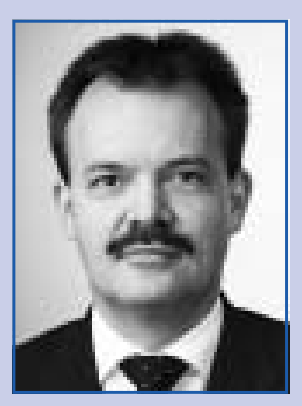

Dr. Matthias Sure ist Dozent für Controlling, Finanzierung und internationales Management an der Hochschule Fresenius in Köln. E-Mail: matthias.sure @hs-fresenius.de

\title{
Working Capital-Steuerung mit integrierten Kennzahlen
}

Matthias Sure

Unsichere und restriktivere Bedingungen bei der externen Kapitalaufnahme führen in Unternehmen häufig zu Finanzierungsproblemen. Eine prozessübergreifende und integrierte Steuerung des Working Capitals kann das Potenzial zur Finanzierung und Liquiditätsgenerierung aus dem eigenen operativen Geschäft heraus stärken. In diesem Beitrag sollen Lösungsansätze zu einer effektiven und integrierten Steuerung des Working Capitals aufgezeigt werden.

\section{Zunehmende Relevanz der Working Capital-Steuerung}

Insbesondere vor dem Hintergrund der im Zuge der Finanzkrise seit einigen Jahren zu beobachtenden steigenden Volatilitäten und Unsicherheiten auf den Kredit- und Finanzmärkten und der damit korrespondierenden zunehmenden Regulatorik im Bereich der externen Kapitalaufnahme hat sich in vielen Ländern eine vorsichtigere, zuweilen restriktivere Kreditvergabepolitik der Finanzinstitute gegenüber ihren Kunden eingestellt.

Demzufolge hat sich der Fokus in den Unternehmen vermehrt auf die unternehmensinterne Finanzierung und Liquiditätsgenerierung aus dem operativen Geschäft heraus und damit auf die Gestaltung und die Steuerung des Working Capitals verlagert. Diese Entwicklung geht einher mit der notwendigen Konsequenz einer stärkeren Integration von Working Capital-bezogenen Kennzahlen in die Unternehmenssteuerung. Dennoch sind in der Praxis umfangreiche Steuerungsmechanismen zum Working Capital in Form von zusammenhängenden Kennzahlen immer noch die Ausnahme. Zielsetzung dieses Beitrags ist es, in diesem Kontext Lösungsansätze zu einer effektiven und integrierten Steuerung des Working Capitals aufzuzeigen. Dazu werden zunächst Gegenstand und Zweck einer Working Capital-Steuerung thematisiert, gefolgt von einer Darstellung damit korrespondierender Kennzahlen, welche schließlich in ein integriertes Kennzahlensystem überführt werden.

\section{Gegenstand und Zweck einer Working Capital-Steuerung}

Grundsätzlich sind zwei Betrachtungsweisen des Working Capitals zu unterscheiden. Das Bruttoumlaufvermögen (Gross Working Capital) verkörpert das gesamte Umlaufvermögen, welches im Unternehmen für das tägliche operative Geschäft zur Verfügung steht. Demgegenüber bezeichnet das Nettoumlaufvermögen (Net Working Capital) den Überschuss, der sich aus der Differenz zwischen Umlaufvermögen und kurzfristigen Verbindlichkeiten ergibt.

Das Net Working Capital kann also einerseits zur Ermittlung der eingetretenen Liquiditätsveränderung und andererseits zur Abschätzung eines vorhandenen langfristigen Finanzierungspotenzials und damit des zukünftigen Liquiditätsrisikos herangezogen werden (vgl. Perridon et al., 2012, S. 604-605). Auch wenn sich bis heute in der Literatur keine allgemeingültige Definition des Begriffs etabliert hat, orientiert sich die Bestimmung des Net Working Capitals in den Unternehmen häufig an der Summe aus den operativen Umlaufvermögen-Positionen Vorräte, Forderungen aus Lieferungen und Leistungen, geleistete Anzahlungen sowie sonstige kurzfristige Forderungen abzüglich der operativen kurzfristigen Verbindlichkeiten-Positionen Verbindlichkeiten aus Lieferungen und Leistungen, erhaltene Anzahlungen, operative Rückstellungen sowie sonstige kurzfristige Verbindlichkeiten (vgl. Maness/Zietlow, 2005, S. 29-30). 


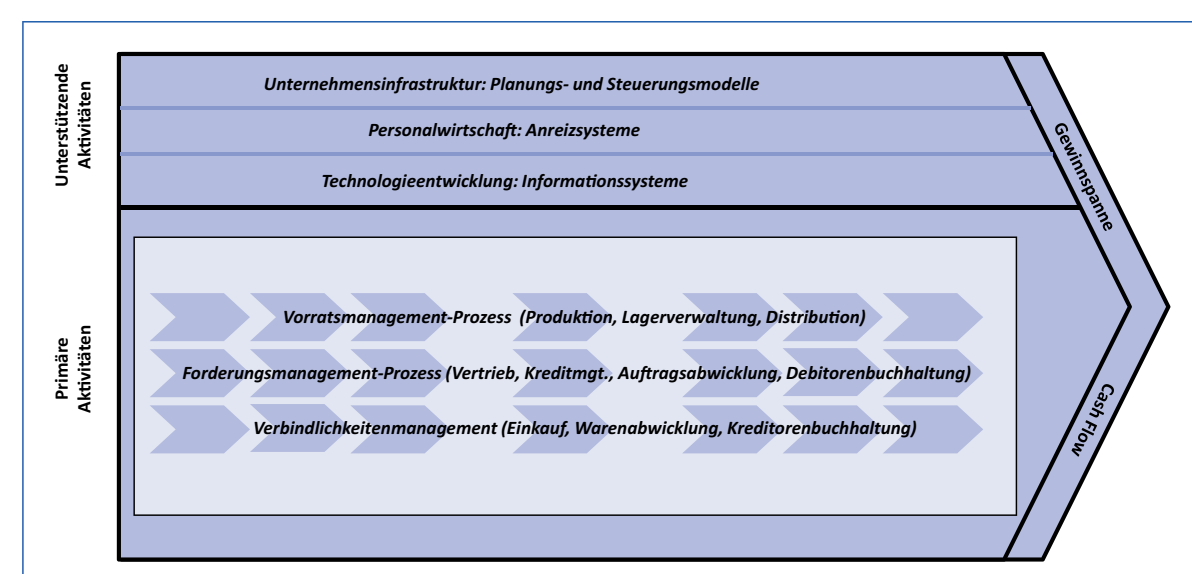

Abb. 1: Working Capital-Prozesse in der Wertschöpfungskette

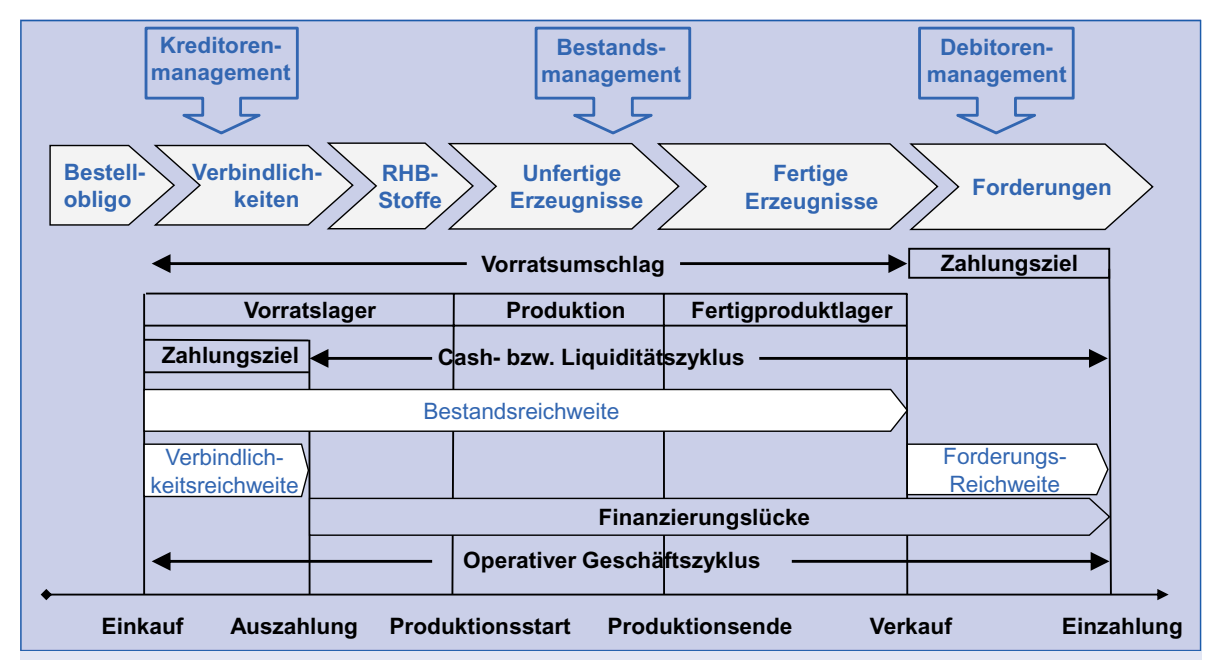

Abb. 2: Operativer Geschäftszyklus und Cash-Zyklus

Der duale Fokus der Working CapitalSteuerung beinhaltet einerseits die Gestaltung des Liquiditäts- und Wertflusses zwischen den unterschiedlichen Working Capital-Positionen und anderen Bilanzpositionen zur Sicherung einer angemessenen Liquidität und andererseits die Gewährleistung erforderlicher Bestände in einzelnen Working Capital-Positionen zur Steigerung der Profitabilität. Aufgabe der Steuerung des Working Capitals ist es daher, im Rahmen der Investition in sowie der Finanzierung von Working Capital eine optimale Balance zwischen Profitabilität und Liquidität bzw. Rentabilität und Risiko vor dem Hintergrund der gesamtunternehmerischen Ziele zu erreichen.

Dabei wird evident, dass die Steuerung des Working Capitals zur Erfüllung dieser Aufgabe sowohl Elemente und Prozesse des operativen Managements - insbesondere der Beschaffung und des Vertriebs als auch des Finanzmanagements ein- schließt und damit integrativ ausgelegt sein muss, wobei zu beachten ist, dass das im Unternehmen vorgehaltene Niveau an Working Capital maßgeblich von den Charakteristika des operativen Geschäftsmodells eines Unternehmens bestimmt wird (vgl. Meyer, 2007, S. 35). Vor dem Hintergrund zunehmender Relevanz der Working Capital-Steuerung kommt der Auswahl geeigneter integrierter Messgrößen eine wichtige Bedeutung zu.

\section{3. Übergreifende Kennzahlen zur Working Capital-Steuerung}

Um die Working Capital-Performance eines Unternehmens analysieren und messen zu können, ist in der Konsequenz $\mathrm{zu}$ vorstehender Argumentation eine mehrdimensionale funktionsübergreifende Betrachtung notwendig, die die drei Komponenten des Working Capitals,
Vorräte, Forderungen und Verbindlichkeiten aus Lieferungen und Leistungen prozessual entlang der Wertschöpfungskette analysiert, was durch die traditionellen eindimensionalen Kennzahlen des Gross bzw. Net Working Capitals als reine Volumensgrößen nicht abbildbar ist (vgl. Abb. 1).

In diesem Kontext lassen sich das Working Capital und die Beziehungen zwischen seinen Komponenten stattdessen adäquat durch den Cash- bzw. Liquiditätszyklus abbilden. Konzept und Betrachtungsweise sind hier dynamischer Natur und fokussieren auf den Fluss von operativen Zahlungsströmen bzw. Cash Flows und die damit verbundene Wertgenerierung (vgl. Eitelwein/Wohlthat, 2005, S. 417). Der Cash-Zyklus ist dynamisch in dem Sinn, dass er Bilanz- und GuVDaten kombiniert, daraus ein Maß mit einer Zeitdimension kreiert und in diesem Zuge eine ganzheitliche, dynamische Betrachtung und Analyse des Working Capitals und der Selbstfinanzierungsfähigkeit entlang der betrieblichen Wertschöpfungskette ermöglicht. Nachteilig am Cash-Zyklus ist dagegen, dass seine zeitraumbezogene Betrachtung nicht ohne Weiteres in korrespondierende Volumina gebundenen Working Capitals überführt werden kann.

Entscheidende Messgröße im Cash-Zyklus (vgl. Abb. 2) ist die Kennzahl Days Working Capital (DWC). Sie repräsentiert die Geschwindigkeit, mit der ein Unternehmen Geldabflüsse in Geldzuflüsse umwandelt und gibt an, wie viele Tage Umsatz im Unternehmen vorfinanziert werden müssen. Die Days Working Capital setzen sich zusammen aus der Forderungsumschlagszeit (Forderungsreichweite) zuzüglich der Vorratsumschlagszeit (Bestandsreichweite) abzüglich der Zahlungsverzögerungszeit (Verbindlichkeitenreichweite), welche im Folgenden näher betrachtet werden.

\section{Kennzahlen zum Forderungsmanagement}

Die Forderungsreichweite (Days Sales Outstanding - DSO) gibt an, wie viele Tage Umsatz im Forderungsbestand enthalten sind und misst die Anzahl der Tage, die vergehen von der Rechnungsstellung an den Kunden bis zum Zahlungseingang (vgl. Schneider, 2002, S. 544). Häufig wird dabei eine Berechnung auf Monatsbasis zu Grunde gelegt, indem der Forderungsbestand am Ende des Be- 
richtsmonats durch den kreditsubventionierten Umsatz des Berichtsmonats dividiert und mit der Anzahl der Tage des Berichtsmonats multipliziert wird. Im Rahmen der Berechnung der Forderungsreichweiten ist allerdings $\mathrm{zu}$ berücksichtigen, dass diese regelmäßig Verzerrungen insbesondere durch umsatzinduzierte Volumensschwankungen unterliegen, welche besonders stark in Branchen mit erheblichen saisonalen Zyklen auftreten, was deren Interpretation und Vergleichbarkeit im Zeitablauf erschwert (vgl. Gallinger/Healey, 1987, S. 338 bzw. 341).

Diesen Mangel versuchen Unternehmen in der Praxis häufig durch eine entsprechende Glättung der Umsatz- und Forderungswerte auf Zwei- oder Dreimonatsbasis oder durch Vergleiche der Forderungsreichweiten mit historischen Werten entsprechender Monate zumindest teilweise zu beheben, ohne damit die Grundproblematik wirklich zu beseitigen. Um Verzerrungen der Forderungsreichweite durch unregelmäßige Umsatzmuster wirksam zu verhindern, können dagegen Kalkulationsmethoden zur Berücksichtigung des tatsächlichen Kundenzahlungsverhaltens eingesetzt werden, um auf diese Weise analysieren $\mathrm{zu}$ können, welcher Umsatzanteil eines bestimmten Monats den Forderungspositionen des gleichen Monats sowie der folgenden Monate zuzuordnen ist (vgl. Klepzig, 2010, S. 68). Eine damit eng zusammenhängende Methode ist die so genannte Ausschöpfungsmethode, mit der gemessen wird, wie viele Tage man vom Berichtszeitpunkt aus zurückgehen muss, damit der Umsatz den Bestand an Forderungen abdeckt bzw. erreicht, deren Umsetzung in der unternehmerischen Praxis allerdings häufig noch an technischer bzw. fachlicher Komplexität scheitert.

Um die Aussagekraft des Days Sales Outstanding-Wertes weiter zu erhöhen, kann dieser durch die Kennzahl Best Possible Days Sales Outstanding (BPDSO) ergänzt werden, denn letztere gibt Auskunft darüber, welche durchschnittlichen Forderungsreichweiten-Kennzahlenwerte im optimalen Fall zu erreichen wären, vorausgesetzt, dass auf der Kundenseite jeweils die Zahlungsfristen und -bedingungen eingehalten werden. So wird es möglich, die auf der Forderungsseite wichtigen Forderungs-Überfälligkeitstage (Days Delinquent Sales Outstanding bzw. DDSO) als Kennzahl auszuweisen (DDSO=DSO-BPDSO), welche besondere Relevanz im Rahmen der Steuerung der Kreditmanagement-Prozesse entfalten (vgl. Ertl, 2004, S. 156).

Eine weitere in diesem Kontext messbare Größe betrifft die Days Billing Outstanding (DBO), mit welcher diejenige Zeitspanne gemessen wird, die vergeht vom Zeitpunkt des konkreten Rechnungserstellungsanlasses bis zu deren tatsächlicher Ausstellung bzw. Versendung an den Kunden (vgl. Döring et al., 2012, S. 412-413). Sie stellt ein Maß für die Effizienz des Rechnungserstellungsprozesses dar und ermöglicht es, Schwachstellen zu identifizieren, die - abgesehen von marktbedingten Ursachen wie Zahlungsschwierigkeiten der Kunden oder schwacher Marktposition des Unternehmens neben Ineffizienzen im Mahn- und Zahlungseingangsprozess $\mathrm{zu}$ den wesentlichen unternehmensintern bedingten Ursachen für lange Forderungsreichweiten zählen. Neben DSO sind jedoch die Forderungsreichweite ergänzende Kennzahlen in der Praxis deutlich unterrepräsentiert. So fehlt häufig der notwendige Kontext für ein zusammenhängendes Ursache-Wirkungsgeflecht von Working Capital-Kennzahlen.

Die Forderungsumschlagshäufigkeit weist als Kehrwert der Forderungsreichweite den Faktor aus, um welchen der Umsatz den Forderungsbestand übersteigt und zeigt an, wie oft sich die Investition des Unternehmens in den Forderungsbestand während der betrachteten Periode in Umsatz umschlägt (vgl. Maness/Zietlow, 2005, S. 207). Demzufolge führt ein steigender Forderungsumschlag zu steigender Liquidität und umgekehrt. Weitere Messgrößen betreffen die Forderungsüberfälligkeitsrate (Overdue Rate), die den Anteil überfälliger, also über den eigentlichen Fälligkeitstermin hinaus bestehender Forderungen am Gesamtforderungsbestand ausweist sowie die Forderungsausfallrate (Bad Debt Rate), die den Betrag der Forderungsausfälle in Form der vorgenommenen Abschreibungen auf uneinbringliche Forderungen in Relation zum Umsatz setzt, womit beide Quoten Aufschluss über die Qualität bzw. Effizienz des Forderungsmanagements geben (vgl. Schneider, 2002, S. 544). Problematisch hinsichtlich der Aussagefähigkeit der Forderungsausfallrate ist jedoch die Tatsache, dass diese von der Abschrei- bungspraxis des jeweiligen Unternehmens nicht unwesentlich beeinflusst werden kann. Trotz solcher Probleme sind diese Kennzahlen in der unternehmerischen Praxis deutlich häufiger präsent als die Cash-Zyklus-Größen, was auch daran $\mathrm{zu}$ liegen scheint, dass Volumensgrößen dort leichter verständlich und vermittelbar sind als zeitbezogene Kennzahlen.

Forderungsreichweite und -umschlagshäufigkeit sowie Forderungsüberfälligkeits- und -ausfallrate werden sinnvollerweise ergänzt durch Altersstrukturlisten, welche auf Forderungseinzelpostenebene Überfälligkeiten nach Laufzeitklassen anzeigen, die typischerweise in 30 Tage-Abschnitte gestaffelt sind. Im Rahmen der Interpretation von Altersstrukturlisten liefern Vergleiche von ausstehenden Forderungen mit entsprechend vereinbarten Zahlungsfristen wertvolle Informationen zum Risikoprofil, da sich das Verlustrisiko mit steigendem Alter einer Forderung überproportional erhöht (vgl. Werdenich, 2008, S. 60). Dabei ist allerdings zu beachten, dass Altersstrukturlisten den gleichen Anfälligkeiten für Informationsverzerrungen unterliegen wie die Forderungsreichweite. Insgesamt ist bei der Kreditüberwachung $\mathrm{zu}$ beachten, dass eine isolierte Betrachtung einzelner Kennzahlen zu wenig substanziellen Ergebnissen führt und diese erst dann maßgeblich an Aussagekraft gewinnen, wenn sie zu einem Kennzahlensystem zusammengeführt werden und dabei ein Vergleich mit Vergangenheits- und Planwerten sowie mit Branchenwerten erfolgt.

\section{Kennzahlen zum Verbindlichkeitenma- nagement}

Die Verbindlichkeitenreichweite (Days Payables Outstanding) gibt an, wie viele Tage ein Unternehmen Lieferantenkredite in Anspruch nimmt und misst die Anzahl der Tage, die von der Rechnungsstellung des Lieferanten bzw. dem Rechnungseingang bis zum Zahlungsausgang vergehen (vgl. Schneider, 2002, S. 544). Analog der Forderungsreichweite wird auch hinsichtlich der Verbindlichkeitenreichweite zuweilen eine Berechnung auf Monatsbasis zu Grunde gelegt, indem der Verbindlichkeitenbestand am Ende des Berichtsmonats durch den Umsatz bzw. die Herstellungskosten der abgesetzten Erzeugnisse des Berichtsmonats dividiert und mit der Anzahl der Tage des Berichtsmonats multipliziert wird. 
Einige Autoren wählen für den Ausweis der Verbindlichkeitenreichweite als Bezugsgröße zum Verbindlichkeitenbestand das tägliche bzw. periodische Einkaufsvolumen teilweise unter Zuhilfenahme der Argumentation, dass dieses die sensiblere Bezugsgröße verglichen mit den Herstellungskosten der abgesetzten Erzeugnisse sei, während sie aber gleichzeitig darauf verweisen, dass die Herstellungskosten der abgesetzten Erzeugnisse wegen ihres obligatorischen Ausweises in der Gewinn- und Verlustrechnung die gängigere Bezugsgröße seien, zumal die Größe des Einkaufsvolumens nicht immer unmittelbar transparent ist und ggfs. für eine Ermittlung zusätzliche Berechnungen in der Form erforderlich sind, als die Herstellungskosten um nicht-einkaufsinduzierte Kosten - wie insbesondere Abschreibungen - bereinigt werden müssen (vgl. Fabozzi et al., 2008, S. 275-276). In der Praxis wird die Verbindlichkeitenreichweite deutlich weniger oft gemessen als die Forderungsreichweite, was auch mit deren geringerer Bedeutung in der wissenschaftlichen Literatur einhergeht.

So wie für die Forderungsreichweitenberechnung ist auch im Rahmen der Berechnung der Verbindlichkeitenreichweiten ins Kalkül zu ziehen, dass diese regelmäßigen Verzerrungen unterliegen können, welche aufgrund von saisonalen oder anderen signifikanten umsatz- bzw. einkaufsbezogenen Volumensschwankungen entstehen und dadurch deren Interpretation sowohl punktuell als auch im Zeitvergleich erschwert wird. Daher wird erst eine Begutachtung der Zahlungsmuster in der Relation zwischen Auszahlungen und Beschaffungsvolumen als eigentlicher Quelle der Zahlungsverpflichtung Klarheit über das tatsächliche Zahlungsverhalten eines Unternehmens bringen (vgl. Meyer, 2007, S. 91). In diesem Zuge ist dann etwa mittels der Ausschöpfungsmethode zu analysieren, welcher Beschaffungsanteil eines bestimmten Monats den Verbindlichkeitenpositionen des gleichen Monats sowie der folgenden Monate zuzuordnen ist.

Darüber hinaus kann entsprechend der dargestellten Methodik im Forderungsmanagement die Aussagefähigkeit des Days Payables Outstanding-Wertes dadurch erhöht werden, dass diesem die Kennzahl Best Possible Days Payables Outstanding (BPDPO) zur Seite gestellt wird, um Informationen darüber zu er- halten, welche durchschnittlichen Verbindlichkeitenreichweiten-Kennzahlenwerte im optimalen Fall unter der Maßgabe zu erreichen wären, dass die Zahlungsfristen durch das bezahlende Unternehmen im Rahmen der mit den Lieferanten vereinbarten Zahlungsbedingungen voll ausgenutzt bzw. eingehalten werden, weswegen diese Kennzahl zuweilen schlicht als durchschnittlich vereinbartes Zahlungsziel (Average Payment Terms) bezeichnet wird (vgl. Metze, 2010, S. 107). Ferner setzt die Verbindlichkeitenumschlagshäufigkeit als Kehrwert der Verbindlichkeitenreichweite je nach Berechnungsart den Umsatz bzw. die Herstellungskosten der abgesetzten Erzeugnisse oder das Beschaffungsvolumen ins Verhältnis zu den Verbindlichkeiten aus Lieferungen und Leistungen und weist dabei den Faktor aus, um welchen der Umsatz bzw. das Beschaffungsvolumen den Verbindlichkeitenbestand übersteigt bzw. letzterer sich umschlägt.

Verbindlichkeitenreichweite und -umschlagshäufigkeit werden für gewöhnlich ergänzt durch Altersstrukturlisten, welche auf Verbindlichkeiteneinzelpostenebene die prozentualen Fälligkeitsanteile nach Laufzeitklassen anzeigen, die analog den Strukturen auf der Forderungsseite typischerweise in 30 Tage-Abschnitte gestaffelt sind. Hinsichtlich der potenziellen Verzerrung von Verbindlichkeitenreichweite und Altersstrukturlisten gilt das bereits zur Forderungsreichweite angemerkte. Eine weitere maßgebliche Kennzahl zur Messung des Zahlungsverhaltens insbesondere zur Vermeidung von Vorfälligkeitszahlungen ohne Skontoziehung ist der Skontoausnutzungsgrad, der den Anteil an tatsächlicher Skonto-Inanspruchnahme im Verhältnis zum von den Lieferanten angebotenen Skonto-Gesamtvolumen darstellt und damit Auskunft über Grad und Höhe der Skontoziehungspraxis im beschaffenden Unternehmen gibt (vgl. Sagner, 2011, S. 135). Hinsichtlich der Kennzahlen zum Verbindlichkeitenmanagement sind insgesamt eine niedrigere Präsenz und ein schwächerer Integrationsgrad in der Praxis festzustellen.

Auch für die Messung und Überwachung der Leistungen im Verbindlichkeitenmanagement gilt, dass einzelne isolierte Kennzahlen in der Regel wenig Aussagekraft bieten können und erst deren Einbettung in ein integriertes Kennzahlensystem mit Vergangenheits- und Planwer- ten sowie mit Branchenwerten als Vergleichsmaßstäben verlässliche Schlussfolgerungen zulässt. Auf dieser Basis durchgeführte Abweichungsanalysen tragen dazu bei, dass Gründe für Varianzen in den Verbindlichkeitsbeständen, die insbesondere in Veränderungen des Zahlungsverhaltens und Veränderungen des Beschaffungsvolumens bestehen können, identifiziert und deren Effekte unterschieden werden können.

\section{Kennzahlen zum Vorratsmanagement}

Die Vorratsbestandsreichweite (Days Inventory Held) gibt an, wie viele Tage Bestände im Vorratsvermögen gebunden sind und misst die Anzahl der Tage, die vom Wareneingang über die Produktion bis zum Warenausgang vergehen sowie damit zugleich die durchschnittliche Reichweite der Lagerbestände. Aus Gründen einer Differenzierung zwischen den unterschiedlichen Vorratsgüterkategorien ist eine separate Ermittlung der Vorratsbestandsreichweiten zu RHB-Stoffen, unfertigen und fertigen Erzeugnissen je nach Art des jeweiligen zu Grunde liegenden Geschäftsmodells und der damit verbundenen Gestalt der Supply Chain sinnvoll, um Optimierungspotenziale besser eingrenzen zu können (vgl. Sagner, 2011, S. 113). Entsprechend der Forderungs- und Verbindlichkeitenreichweite wird ebenso hinsichtlich der Vorratsbestandsreichweite - insbesondere bei substanziellen Schwankungen der Vorratsbestände im Verlauf eines Jahres - zuweilen eine Berechnung auf Monatsbasis zu Grunde gelegt, indem der Vorratsbestand am Ende des Berichtsmonats durch den Umsatz bzw. die Herstellungskosten der abgesetzten Erzeugnisse des Berichtsmonats dividiert und mit der Anzahl der Tage des Berichtsmonats multipliziert wird.

Analog zur Ermittlung der Verbindlichkeitenreichweite wählen einige Autoren auch für den Ausweis der Vorratsbestandsreichweite als Bezugsgröße zum Vorratsbestand den Umsatz der Periode, wobei allerdings zu berücksichtigen ist, dass damit der Kennzahlenwert niedriger ausfällt als im Falle des Ansatzes von Herstellungskosten der abgesetzten Erzeugnisse. Für die Berücksichtigung von Herstellungskosten der abgesetzten Erzeugnisse anstelle des Umsatzes spricht außerdem die Tatsache, dass aufgrund vorherrschender IFRS-Rechnungslegungsvorschriften Vorräte mit ihren Anschaf- 


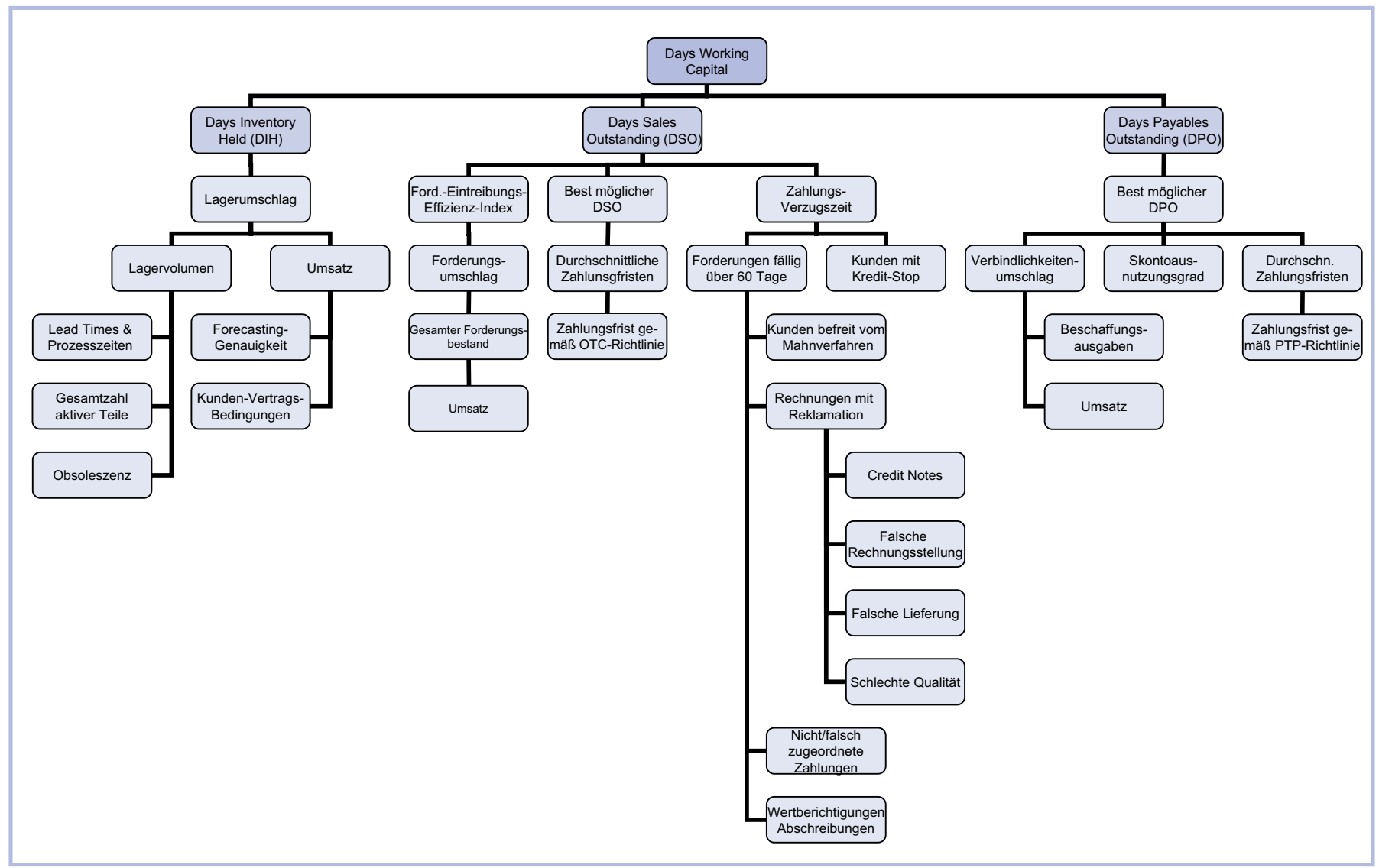

Abb. 3: Integriertes Working Capital-Kennzahlensystem

fungs- oder Herstellungskosten vermindert um Abschreibungen, aber ohne Einbezug von allgemeinen Verwaltungsgemeinkosten und Vertriebskosten anzusetzen sind (vgl. Pellens et al., 2011, S. 411415).

Um Verzerrungen bezüglich des Vorratsbestandsreichweitenausweises aufgrund von saisonalen oder anderen signifikanten Schwankungen der Umsatz- bzw. Einkaufsvolumina wirksam begegnen zu können, können Kalkulationsmethoden wie die Ausschöpfungsmethode zur Ermittlung des tatsächlichen Verbrauchsverhaltens eingesetzt werden, die Analysen ermöglichen, welcher Umsatz- oder Herstellungskostenanteil eines bestimmten Monats mit den Vorratsbestandspositionen des gleichen Monats sowie der folgenden Monate korrespondiert (vgl. Klepzig, 2010, S. 68). Daneben existieren im Bereich des Vorratsvermögens Kennzahlen zur Ermittlung des durchschnittlich im Lagerbestand gebundenen Kapitals etwa in Form der Lager- bzw. Vorratsumschlagshäufigkeit. Diese wird berechnet als Relation zwischen den Her- stellungskosten der abgesetzten Erzeugnisse und dem durchschnittlichen Vorratsbestand. Sie gibt damit an, wie oft die Vorräte durchschnittlich durch die Wertschöpfungskette des Unternehmens in einer Periode hindurchfließen (vgl. Fabozzi et al., 2008, S. 605). Der reziproke Charakter dieser Kennzahl in Relation zur Vorratsbestandsreichweite wird dadurch offensichtlich, dass mit steigender Vorratsumschlagshäufigkeit die Vorratsbestandsreichweite abnimmt und umgekehrt.

Die Vorratsumschlagshäufigkeit kann darüber hinaus als Indikator ergänzt werden durch die Kennzahl Teilezahl, die Auskunft über die Anzahl an Teilen, Komponenten, Zwischen- oder Endprodukten im Lager und damit zusätzliche Informationen zur Kapitalbindung im Vorratsvermögen sowie zu dessen Komplexität, die zugleich maßgeblich von der Sortimentsgestaltung geprägt ist, gibt (vgl. Schneider, 2003, S. 316). Ferner liefern Kennzahlen zur Obsoleszenz etwa in Form der Relation zwischen betragsund/oder mengenmäßiger Vorratsgüter-
Obsoleszenz und dem durchschnittlichen wert- bzw. mengenmäßigen Vorratsbestand ergänzende Aussagen über den Einfluss nicht realisier- bzw. absetzbarer Bestände auf Sicherheitsbestände sowie die Effektivität und Kapitalbindung des Vorratsmanagementprozesses. Häufig mangelt es an der Integration von Kennzahlen zum Vorratsmanagement in der Praxis auch deswegen, weil dessen Steuerung zumeist in der Verantwortung der Supply-Chain-Abteilungen liegt und nicht in der Finanzabteilung wie beim Forderungs- und Verbindlichkeitenmanagement.

Ferner ist dazu anzumerken, dass je nach Geschäfts- und Supply Chain-Modell neben dem operativen Vorratsmanagementprozess auch die Vorratsbewertung maßgeblichen Einfluss auf wichtige Bilanzgrößen und damit auf Kennzahlenausprägungen haben kann.

\section{Zusammenführung zu einem integrierten Kennzahlensystem}

Eine auf den vorstehend beschriebenen Kennzahlen basierende integrierte und 
mehrdimensionale Steuerung des Working Capitals kann durch ein Kennzahlensystem reflektiert und unterstützt werden, wie das Beispiel in Abb. 3 aus der Unternehmenspraxis zeigt. Dieses Kennzahlensystem wurde in einem Unternehmen mittels funktionsübergreifender Workshops unter Federführung des Finanzdirektors über mehrere Monate entwickelt sowie in ein spezielles Working Capital-Reporting überführt und trägt seitdem maßgeblich zu einer verbesserten Liquiditätsgenerierung und Finanzierung aus eigener Kraft bei, infolge deren die Abhängigkeit von der externen Bankenfinanzierung reduziert werden konnte. Dabei wurde besonderer Wert auf die angesprochene Kombinatorik aus zeitraumund volumensbasierten Kennzahlen gelegt und ausreichend Zeit eingeräumt, um die für die operativen Einheiten nicht immer leicht verständlichen zeitraumbezogenen Größen des Cash-Zyklus ausreichend zu erläutern sowie die notwendige Akzeptanz für diese in der Organisation zu schaffen.

In einem solchen report-unterstützten Kennzahlensystem werden die das Working Capital beeinflussenden Treiber und deren Ursache-Wirkungsbeziehungen transparent und damit für alle Beteiligten im Unternehmen in ihren Auswirkungen sichtbar und verständlich, sodass eine übergreifende Working Capital-Steuerung auf Basis von zeitraum- und volumensbasierten Größen möglich wird.

\section{Fazit}

Die Steuerung des Working Capitals nimmt in der unternehmerischen Praxis einen zunehmend größeren Stellenwert ein, was unter anderem aus volatileren und schwierigeren Bedingungen auf den Kreditmärkten resultiert. Dabei nehmen zeitraumbezogene Steuerungsgrößen wie der Cash-Zyklus an Bedeutung zu. Um eine Steigerung der Qualität im Working Capital Management zu erzielen, wird es zukünftig für die Unternehmen auf eine stärkere Fokussierung zeitraumbezogener Steuerungsgrößen, auf deren sinnvolle
Verzahnung mit volumensorientierten Größen im Sinne stärker integrierter Working Capital-bezogener Kennzahlensysteme sowie auf deren adäquate Abbildung in einem Working Capital-Berichtswesen ankommen.

Keywords
Cash Conversion Cycle
Days Inventory Held
Days Payables Outstanding
Days Sales Outstanding
Working Capital
Summary
Due to credit market volatilities and
difficulties in obtaining external loans,
working capital performance mea-
surement has become an area of focus
in corporate management. In order to
improve working capital performance,
cross-functional time-based perfor-
mance measures would need to be ac-
cordingly combined with volume-
based ones. The purpose of this article
is to demonstrate possible solutions to
an effective and integrated working
capital management.

\section{Literatur}

Döring, O./Schönherr, M./Steinhäuser, P., Working Capital Controlling - Entwicklung eines Steuerungskonzepts zur nachhaltigen Performancesteigerung eines mittelständischen Unternehmens, in: Controlling - Zeitschrift für erfolgsorientierte Unternehmenssteuerung, 24. Jg. (2012), H. 8/9, S. 409-415.

Eitelwein, O./Wohlthat, A., Steuerung des Working Capital im Supply Chain Management über die Cash-to-Cash Cycle Time, in: Zeitschrift für Controlling \& Management, 49. Jg. (2005), H. 6, S. 416-425.

Ertl, M., Aktives Cashflow-Management - Liquiditätssicherung durch wertorientierte Unternehmensführung und effiziente Innenfinanzierung, München 2004.

Fabozzi, F. J./Drake, P. P./Polimeni, R. S., The Complete CFO Handbook - From Accounting to Accountability, New Jersey u. a 2008.

Gallinger, G. W./Healey, P. B., Liquidity Analysis and Management, Reading, Massachusetts u. a 1987.
Klepzig, H., Working Capital und Cash Flow - Finanzströme durch Prozessmanagement optimieren, 2. Aufl., Wiesbaden 2010.

Maness, T. S./Zietlow, J. T., Short-Term Financial Management, 3. Aufl., Mason 2005.

Metze, T., Supply Chain Finance - Die Wertorientierte Analyse und Optimierung des Working Capital in Supply Chains, Diss., Köln 2010.

Meyer, C. A., Working Capital und Unternehmenswert, Diss., Wiesbaden, 2007.

Pellens, B./Fülbier, R. U./Gassen, J./Sellhorn, T., Internationale Rechnungslegung, 8. Aufl., Stuttgart 2011.

Perridon, L./Steiner, M./Rathgeber, A., Finanzwirtschaft der Unternehmung, 16. Aufl., München 2012.

Prasad, B./Eresi, K., Working Capital Management in SSI - An Empirical Study, in: Tayal, P. K./Jain, S. C., Management of Working Capital, Jaipur 2009, S. 38-49.

Sagner, J. S., Essentials of Working Capital Management, New Jersey 2011.

Schneider, C., Controlling von Working Capital bei Logistikdienstleistern, in: Controller Magazin, 27. Jg. (2002), H. 6, S. 540-546.

Schneider, C., Working Capital Management, in: wisu - das wirtschaftsstudium, 32. Jg. (2003), H. 3, S. 315-318.

Werdenich, M., Modernes Cash-Management - Instrumente und Maßnahmen zur Sicherung und Optimierung der Liquidität, 2. Aufl., München 2008.

Literaturtipps aus dem Online-Archiv der ConTROLLING:

- Reinhard Rupp, Working Capital Management: Controlling mit eindrucksvollen Bildern oder mit belastbaren Zahlen?, Ausgabe 7/2011, S. 379-386.

- Teresa Langer und Marcus Schubbe, Working Capital Management und Controlling im internationalen Stahlhandel, Ausgabe 7/2010, S. 368-374.

- Matthias Sure, Kosten- und Liquiditätsoptimierung durch integriertes Working Capital Management, Ausgabe 7/2004, S. 393-398. 